PROCEEDINGS OF THE

AMERICAN MATHEMATICAL SOCIETY

Volume 127, Number 8, Pages 2249-2258

S 0002-9939(99)04693-6

Article electronically published on April 16, 1999

\title{
BRAIDED TENSOR C*-CATEGORIES, HECKE SYMMETRIES AND ACTIONS ON EXTENDED CUNTZ ALGEBRAS
}

\author{
ANNA PAOLUCCI
}

(Communicated by Palle E. T. Jorgensen)

\begin{abstract}
In this paper we deal with braided tensor $\mathrm{C}^{*}$-categories. For every object $\rho$ of the category we associate a $\mathrm{C}^{*}$-algebra denoted by $O_{\rho}$. An analysis of the braiding is carried out by using the conjugate equations. If the braiding is a Hecke symmetry and the $q$-dimension is appropriately chosen, we characterize the $\mathrm{C}^{*}$-algebra as the one generated by the representation given by the Markov trace. This analysis leads to the existence of an action of $\mathcal{F}_{S U_{q}(d)}$ on $O_{\rho}$. Such actions (Theorem 1) correspond to *-monomorphisms of $\left(O_{N}\right)^{S U_{q}(d)}$ on $O_{\rho}$ which generalize the ones obtained earlier by the author (1997).
\end{abstract}

\section{INTRODUCTION}

The results of this paper are motivated by earlier works on coactions on Cuntz algebra and the abstract duality theory for compact groups where the basic results rest on endomorphisms of $\mathrm{C}^{*}$-algebras. The paper is organized as follows.

The class of categories studied here is presented in section 2. They are $\mathrm{C}^{*}$ categories, the categorical analogue of $\mathrm{C}^{*}$-algebras equipped with additional structures. There is a monoidal structure which models the tensor product. This structure is braided to model the non-commutativity of the tensor product. We assume also that the $\mathrm{C}^{*}$-categories have conjugates.

We associate to each object in a braided tensor $\mathrm{C}^{*}$-category a $\mathrm{C}^{*}$-algebra $O_{\rho}$ acted upon by an endomorphism $\hat{\rho}$. We discuss also the action of a braided tensor $\mathrm{C}^{*}$-category on the $\mathrm{C}^{*}$-algebra $O_{\rho}$. In section 3 we consider some properties of the braided tensor $\mathrm{C}^{*}$-categories with conjugates. We establish the existence of a q-dimension function on the object of our category. We relate it with the qantisymmetric tensors and the existence of conjugates. If the braiding satisfies the Hecke symmetry conditions, then we prove that a Markov trace exists for a specific choice of the q-dimension and in the theorem we give the characterization of the $\mathrm{C}^{*}$ algebra generated by the Hecke symmetry. In the last section we prove the existence of a ${ }^{*}$-monomorphism into the extended Cuntz algebra $O_{\rho}$ uniquely defined by the Hecke symmetry and a determinant $R \in\left(i, \rho^{N}\right)$ satisfying the conjugate property of Lemma 4. We also use the recent Theorem 3.1 of [1] which associates a compact matrix quantum group to a Hecke symmetry of rank $N$. This theorem is used in

Received by the editors July 22, 1997 and, in revised form, August 11, 1997, August 18, 1997, and September 11, 1997.

1991 Mathematics Subject Classification. Primary 46M05, 16W30, 81R50.

Key words and phrases. $\mathrm{C}^{*}$-algebras, Hilbert spaces, Hecke symmetries, braided $\mathrm{C}^{*}$-categories.

(C)1999 American Mathematical Society 
a crucial way to get the abstract tensor $\mathrm{C}^{*}$-category associated to the one of the universal compact quantum group $S U_{q}(N)$. Our result in the theorem extends the result of [9] to braided $\mathrm{C}^{*}$-categories with conjugates and is related to the results obtained by [3] for the case of symmetric tensor $\mathrm{C}^{*}$-categories.

\section{BASIC DEFINITIONS}

Let $\mathcal{F}$ be a category whose objects we denote by $\rho, \sigma, \tau, \ldots$ [6], [8]. The set of arrows between a pair $\rho, \sigma$ of objects will be denoted by $(\rho, \sigma)$ and the identity of $\rho$ by $1_{\rho}$. Then $\mathcal{F}$ will be called a $\mathrm{C}^{*}$-category if each $(\rho, \sigma)$ is a complex Banach space where the composition of arrows gives us a bilinear map $S, R \longrightarrow$ $S \circ R$, with $\|S \circ R\| \leq\|S\|\|R\|$ and there is an antilinear involutive contravariant functor ${ }^{*}: \mathcal{F} \longrightarrow \mathcal{F}$ where if $R \in(\rho, \sigma)$, then $R^{*} \in(\sigma, \rho)$ with $\left\|R^{*} \circ R\right\|=\|R\|^{2}$.

A $\mathrm{C}^{*}$-category with a single object is just a $\mathrm{C}^{*}$-algebra with unit. $\mathcal{F}$ is said to have subobjects if given a (self-adjoint) projection $E \in(\rho, \rho)$ there is an isometry $V \in(\sigma, \rho)$ with $V \circ V^{*}=E$. $\mathcal{F}$ is said to have (finite) direct sums if given objects $\rho, \sigma$ of $\mathcal{F}$ there are isometries $V \in(\rho, \tau), W \in(\sigma, \tau)$ with $V \circ V^{*}+W \circ W^{*}=1_{\tau}$.

We introduce further structure on a $\mathrm{C}^{*}$-category, modelled on the behavior of tensor products. $\mathcal{F}$ is said to be a strict monoidal (or tensor) $\mathrm{C}^{*}$-category if the following holds. To each pair $\rho, \sigma$ of objects of $\mathcal{F}$ there is a product object denoted simply by $\rho \sigma$ and $\mathcal{F}$ contains a unit object $i$ such that $\rho i=i \rho=\rho$. Given two arrows $R \in(\rho, \sigma)$ and $R^{\prime} \in\left(\rho^{\prime}, \sigma^{\prime}\right)$ there is an arrow $R \times R^{\prime} \in\left(\rho \rho^{\prime}, \sigma \sigma^{\prime}\right)$. The mapping $R, R^{\prime} \longrightarrow R \times R^{\prime}$ is associative and bilinear and we have $1_{i} \times R=R \times 1_{i}=R$ and $\left(R \times R^{\prime}\right)^{*}=R^{*} \times R^{\prime *}$ and the interchange law $(S \circ R) \times\left(S^{\prime} \circ R^{\prime}\right)=\left(S \times S^{\prime}\right) \circ$ $\left(R \times R^{\prime}\right)$ holds whenever the left hand side is defined. For $R \in(\rho, \sigma), R^{\prime} \in\left(\rho^{\prime}, \sigma^{\prime}\right)$, from this interchange law, $R \times R^{\prime}=\left(1_{\sigma} \times R^{\prime}\right) \circ\left(R \times 1_{\rho^{\prime}}\right)=\left(R \times 1_{\sigma^{\prime}}\right) \circ\left(1_{\rho} \times R^{\prime}\right)$. Note that $(i, i)$ is an abelian $\mathrm{C}^{*}$-algebra.

Thus a strict monoidal (or tensor) $\mathrm{C}^{*}$-category is a $\mathrm{C}^{*}$-category where we have an associative bilinear bifunctor $\mathcal{F} \times \mathcal{F} \longrightarrow \mathcal{F}$ with a unit $i$ and commuting with *.

We now introduce another element of structure on the strict monoidal $\mathrm{C}^{*}$ category which models the non-commutativity of the tensor product, the braiding. We say that $(\mathcal{F}, \theta)$ is a strict braided tensor $\mathrm{C}^{*}$-category if the following conditions are satisfied: to each pair $\rho, \sigma$ of objects of $\mathcal{F}$ there is an operator $\theta(\rho, \sigma) \in(\rho \sigma, \sigma \rho)$ such that if $R \in(\rho, \sigma), R^{\prime} \in\left(\rho^{\prime}, \sigma^{\prime}\right)$, then we have

$$
\theta\left(\sigma, \sigma^{\prime}\right) \circ R \times R^{\prime}=R^{\prime} \times R \circ \theta\left(\rho, \rho^{\prime}\right),
$$

$\theta(\rho, \sigma)$ is natural in $\rho$ and $\sigma$ and

1. $\theta(\rho, i)=\theta(i, \rho)=1_{\rho}$;

2. $\theta(\rho, \sigma \tau)=1_{\sigma} \times \theta(\rho, \tau) \circ \theta(\rho, \sigma) \times 1_{\tau}$;

3. $\theta(\rho \sigma, \tau)=\theta(\rho, \tau) \times 1_{\sigma} \circ 1_{\sigma} \times \theta(\sigma, \tau)$.

This structure is related to the braid group in the following way. As described in [10] given an object $\rho$ in a strict braided tensor category there is a unique braided tensor functor $\theta_{\rho}$ from the braid category $\mathcal{B}$ to $\mathcal{F}$ with $\theta_{\rho}(1)=\rho$. The braiding of $\mathcal{B}$ is determined by $\theta(1,1)$ and we can take $\theta(1,1)=g_{0}$ to be a generator of the braid group of order 2 . Since $\theta_{\rho}$ is braided, then $\theta_{\rho}\left(g_{0}\right)=\theta(\rho, \rho)$. The operation of tensoring on the right by 1 gives the infinite braid group $\mathcal{B}_{\infty}$ as an inductive limit and the endomorphism $\sigma$, which shifts a braid to the left, corresponds to tensoring 
on the left by 1 . We will use this at the end of the section. Let us include here a few basic definitions on Hecke symmetry which will be needed later [16], [1].

Let $q \in \mathbf{C}^{*}$ be such that $q=1$ or $q$ is not a root of unit. Set $[m]_{q}=\frac{q^{m}-q^{-1}}{q-q^{-1}}$, $[m]_{q} !=[1]_{q}[2]_{q} \ldots[m]_{q}$. The Hecke algebra $H_{n}(q)$ is generated by $1, T_{1}, \ldots, T_{n-1}$ and relations $T_{i} T_{j}=T_{j} T_{i}$, if $|i-j|>1 ; T_{i} T_{i+1} T_{i}=T_{i+1} T_{i} T_{i+1}$, if $1 \leq i \leq n-1$; $\left(T_{i}-q\right)\left(T_{i}+1\right)=0$ for $1 \leq i<n$, where 1 is the unit of $H_{n}(q)$. Let $S_{n}$ be the symmetric group on $n$ generators. For any element $\sigma \in S_{n}$ an element $T_{\sigma} \in H_{n}(q)$ can be defined as follows. Choose $\sigma \in S_{n}$ as the product of elementary transposition $\sigma_{i}=(i, i+1) \in S_{n}, \sigma=\sigma_{i_{1}} \ldots \sigma_{i_{l}}$, that has the shortest length $l(\sigma)$ and define $T_{\sigma}=$ $T_{i_{1}} \ldots T_{i_{l}}$, where $T_{\sigma}$ does not depend on a choice of a shortest decomposition, and for $m, n \in \mathbf{N}$ there is a morphism of algebras $H_{m}(q) \times H_{n}(q) \longrightarrow H_{m+n}(q)$ given by $\left(T_{i}, 1_{n}\right) \longmapsto T_{i}, 1 \leq i<m ;\left(1_{m}, T_{j}\right) \longmapsto T_{m+j}, 1 \leq j<n$. Let $\sigma_{m, n} \in S_{m+n}$ be the permutation given by $i \longmapsto i+n$ if $1 \leq i \leq m$ and by $i \longmapsto i-m$ if $m+1 \leq i \leq m+n$. Define $S_{m, n}=(-1)^{m n} T_{\sigma_{m, n}} \in H_{m+n}(q)$. It is a braiding. Let $Y_{k}$ be the set of Young diagrams having $k$ boxes, and let $\left\{e_{\lambda}\right\}_{\lambda \in Y_{k}}$ be the set of central minimal idempotents as in [1], [16]. The parity idempotent $e_{k}=$ $\left([k]_{q^{-1}} !\right)^{-1} \sum_{\sigma \in S_{k}}(-q)^{-l(\sigma)} T_{\sigma}$ is the one corresponding to the column having $k$ boxes. A Hecke symmetry is a linear operator $R$ on $H \otimes H$ ( $H$ is a Hilbert space of finite-dimension) satisfying

1. Yang-Baxter equation $R_{1} R_{2} R_{1}=R_{2} R_{1} R_{2}$;

2. Hecke condition $(R-q)(R+1)=0$.

For every $k$ Hecke symmetry defines a representation $\pi_{k}: H_{k}(q) \longrightarrow L\left((H)^{\otimes k}\right)$ by $T_{i} \longmapsto i d_{i-1} \otimes R \otimes i d_{k-i-1}, i d_{m}=i d_{H \otimes m}$. The projection $\pi_{k}\left(e_{k}\right)$ will be denoted by $P_{k}$. The linear space $\Lambda^{k}=\operatorname{Im}\left(P_{k}\right)$ can be thought of as an analogue of the space of antisymmetric tensors in $(H)^{\otimes k}$. It is known that the central minimal idempotents in $H_{k}(q)$ having non-zero weight with respect to the Markov trace of modulus $q^{N}[N]_{q}^{-1}$ are exactly the ones corresponding to Young diagrams having a number of rows not exceeding $N$ so that $P_{N+1}=0, P_{N} \neq 0, \operatorname{rank}\left(P_{N}\right)=1$. Thus by Theorem 3.1 of [1] the $\mathrm{C}^{*}$-algebra $A(R)=C^{*}\left\{u_{i j}, i, j=1, \ldots, n, u\right.$ unitary, $\left.u^{\otimes N} \xi=\xi\right\}$, where $\xi$ is a vector of norm one in $\operatorname{Im}\left(P_{N}\right)$, is a compact matrix pseudogroup [11].

The final element of structure on the category $\mathcal{F}$ concerns the existence of conjugates. Given an object $\rho$ of $\mathcal{F}$ there is an object $\bar{\rho}$ of $\mathcal{F}$ and an $R \in(i, \bar{\rho} \rho)$ such that

$$
\bar{R}^{*} \times 1_{\rho} \circ 1_{\rho} \times R=1_{\rho}, \quad R^{*} \times 1_{\bar{\rho}} \circ 1_{\bar{\rho}} \times \bar{R}=1_{\bar{\rho}}
$$

where $\bar{R}=\theta(\bar{\rho}, \rho) \circ R$. The equations (2) are called conjugate equations. Thus $(\mathcal{F}, \theta)$ is a strict braided tensor $\mathrm{C}^{*}$-category with conjugates [8], [3].

Example. The $\mathrm{C}^{*}$-category of finite-dimensional Hilbert spaces in a $\mathrm{C}^{*}$-algebra $\mathcal{B}$ with unit is a strict tensor $\mathrm{C}^{*}$-category with conjugates where each element is self-conjugate.

Associated to an object $\rho$ in the strict braided tensor $\mathrm{C}^{*}$-category is a $\mathrm{C}^{*}$-algebra $O \rho$. Suppose that the operation of tensoring on the right by 1 is an isometry. For every $k \in \mathbf{Z}$ we have an inductive system of Banach spaces

$$
\left(\rho^{r}, \rho^{k+r}\right) \longrightarrow\left(\rho^{r+1}, \rho^{k+r+1}\right) \longrightarrow\left(\rho^{r+2}, \rho^{k+r+2}\right) \longrightarrow \ldots,
$$

$r \in \mathbf{N}_{0}$, such that $k+r \geq 0$ and mappings are given by tensoring on the right by $1_{\rho}$. Define $O_{\rho}^{k}$ to be the Banach space inductive limit of this system. Note 
that $O_{\rho}^{k *}=O_{\rho}^{-k}$, and $O_{\rho}^{0}$ being an inductive limit of $\mathrm{C}^{*}$-algebras is itself a $\mathrm{C}^{*}$ algebra. The ${ }^{*}$-operation survives the passage to the inductive limit and so does the composition. In fact if $T \in\left(\rho^{r}, \rho^{k+r}\right), S \in\left(\rho^{k+r}, \rho^{j+k+r}\right)$, then $S \circ T \in\left(\rho^{r}, \rho^{j+k+r}\right)$ and $(S \circ T) \times 1_{\rho}=S \times 1_{\rho} \circ T \times 1_{\rho},\|S \circ T\| \leq\|S\|\|T\|$. Hence composition induces a bounded linear mapping $O_{\rho}^{j} \times O_{\rho}^{k} \longrightarrow O_{\rho}^{j+k}$ written as $S, T \longrightarrow S \circ T$. Also $(S \circ T)^{*}=T^{*} \circ S^{*}, S^{*} \circ S \in O_{\rho}^{0}$ and $\left\|S^{*} \circ S\right\|=\|S\|^{2}$. If we want to distinguish between $T \in\left(\rho^{k}, \rho^{k+r}\right)$ and $T$ as an element of $O_{\rho}^{k}$, we will denote the latter as $i(T) .\left\{O_{\rho}^{k}\right\}_{k \in \mathbf{Z}}$ is a $\mathbf{Z}$-graded $\mathrm{C}^{*}$-algebra, i.e. each $O_{\rho}^{k}$ is a Banach space in which the norm satisfies the $\mathrm{C}^{*}$-condition. Thus $O_{\rho}^{0}$ is a $\mathrm{C}^{*}$-algebra and the $\mathrm{C}^{*}$-norm on $O_{\rho}^{k}, k \in \mathbf{Z}$, is unique.

Denote by $O_{\rho}$ the $\mathrm{C}^{*}$-algebra completion of $\bigoplus_{k \in \mathbf{Z}} O_{\rho}^{k}$ with respect to the unique $\mathrm{C}^{*}$-norm. $O_{\rho}$ also has an endomorphism $\hat{\rho}$ constructed as follows: since $1_{\rho} \times$ $\left(T \times 1_{\rho}\right)=\left(1_{\rho} \times T\right) \times 1_{\rho}$, the map $1_{\rho} \times T$ is compatible with the inductive system. Thus there exists an endomorphism $\hat{\rho}$ of $O_{\rho}^{k}$ for which $\hat{\rho}(i(T))=i\left(1_{\rho} \times T\right), T \in$ $\left(\rho^{r}, \rho^{k+r}\right)$. Since $1_{\rho} \times(S \circ T)=1_{\rho} \times S \circ 1_{\rho} \times T$ and $1_{\rho} \times S^{*}=\left(1_{\rho} \times S\right)^{*}$, the map $S \longrightarrow \hat{\rho}(S)$ is an endomorphism of the graded $\mathrm{C}^{*}$-algebra $\left\{O_{\rho}^{k}\right\}_{k \in \mathbf{Z}}$. We thus have an endomorphism $\hat{\rho}$ of $\bigoplus_{k \in \mathbf{Z}} O_{\rho}^{k}$ and since $\|\cdot\|^{\prime}: T \longmapsto \sup \{\|T\|,\|\hat{\rho}(T)\|\}$ is a $\mathrm{C}^{*}$ norm on $\bigoplus_{k \in \mathbf{Z}} O_{\rho}^{k}$, then $\|\hat{\rho}(T)\| \leq\|T\|$ and $\hat{\rho}$ extends to give an endomorphism $\hat{\rho}$ of $O_{\rho}$. Thus as in [3] there is a $\mathrm{C}^{*}$-algebra $O_{\rho}$ and a strict tensor *-functor $i: \mathcal{F}_{\rho} \longrightarrow E n d O_{\rho}$. Suppose that tensoring on the right with $1_{\rho}$ is an isometry in $\mathcal{F}_{\rho}$; then $i$ is faithful. If $\rho$ is an object of a strict braided tensor $\mathrm{C}^{*}$-category $(\mathcal{F}, \theta)$ by the functoriality of this construction, we have that there exists a canonical representation $\theta$ of $\mathcal{B}_{\infty}$ in $O_{\rho}$ with $\theta\left(g_{0}\right)=i(\theta(\rho, \rho))$ and

$$
\begin{gathered}
\theta(\sigma g)=\hat{\rho}(\theta(g)), \\
\theta(g) \hat{\rho}^{n}(A)=\hat{\rho}^{n}(A) \theta(g),
\end{gathered}
$$

where $A \in O_{\rho}, g \in \mathcal{B}_{\infty}$. Note that if we denote the image of the braid that interchanges $r$ with $s$ by $\theta(r, s)$, then $\theta(r, s)=i\left(\theta\left(\rho^{r}, \rho^{s}\right)\right)$.

We say that an endomorphism $\rho$ of a $\mathrm{C}^{*}$-algebra $\mathcal{A}$ with unit has a braiding $\theta$ if we can find such a representation with

1. $\theta\left(\sigma g_{i}\right)=\rho\left(\theta\left(g_{i}\right)\right), g_{i} \in \mathcal{B}_{\infty}$;

2. $\theta(g) \rho^{n}(A)=\rho^{n}(A) \theta(g), \quad A \in \mathcal{A}, g \in \mathcal{B}_{n} \subset \mathcal{B}_{\infty}$;

3. $\theta\left(g_{s}\right) X=\rho(X) \theta\left(g_{r}\right), X \in\left(\rho^{r}, \rho^{s}\right), r, s \in \mathbf{N}_{0}$.

\section{QUANTUM DIMENSION AND BRAIDING}

In this section we establish the existence of a quantum dimension function in a strict braided tensor $\mathrm{C}^{*}$-category with conjugates and relate it to a particular strict tensor braided $\mathrm{C}^{*}$-category. Let $(\mathcal{F}, \theta)$ be a strict braided tensor $\mathrm{C}^{*}$-category with conjugates. We assume that $\mathcal{F}$ contains subobjects and direct sums. The concept underlying the notion of conjugation is that of adjoint functor. Assume $(i, i)=\mathbf{C}$, i.e. $i$ is irreducible.

Definition. Define a left inverse $\phi$ for an object $\rho$ in $\mathcal{F}$ to be the set

$$
\phi_{\sigma, \tau}:(\rho \sigma, \rho \tau) \longrightarrow(\sigma, \tau), \text { for } \sigma, \tau \text { objects of } \mathcal{F}
$$

of linear mappings which are natural in $\sigma$ and $\tau$, i.e. for $S \in\left(\sigma, \sigma^{\prime}\right), T \in\left(\tau, \tau^{\prime}\right)$ we have

$$
\phi_{\sigma^{\prime}, \tau^{\prime}}\left(\left(1_{\rho} \times T\right) \circ X \circ\left(1_{\rho} \times S^{*}\right)\right)=T \circ \phi_{\sigma, \tau}(X) \circ S^{*}, \quad X \in(\rho \sigma, \rho \tau),
$$


and for each object $\pi$ of $\mathcal{F}$ the left inverse $\phi$ also satisfies $\phi_{\sigma \pi, \tau \pi}\left(X \times 1_{\pi}\right)=$ $\phi_{\sigma, \tau}(X) \times 1_{\pi}, X \in(\rho \sigma, \rho \tau)$.

We say that $\phi$ is positive if $\phi_{\sigma, \sigma}$ is positive for each $\sigma$, and normalized if $\phi_{i, i}\left(1_{\rho}\right)=$ 1. $\phi$ is faithful if $\phi_{\sigma, \sigma}$ is faithful for each object $\sigma$. If $\rho$ has a conjugate, then we may always construct non-zero left inverses. Suppose $R, S \in(i, \bar{\rho} \rho)$ and define $\phi_{\sigma, \tau}(X)=S^{*} \times 1_{\tau} \circ 1_{\bar{\rho}} \times X \circ R \times 1_{\sigma}, X \in(\rho \sigma, \rho \tau)$. Then $\phi$ is a left inverse. It is positive if $R=S$ and normalized if $S^{*} \circ R=1_{i}$. Observe that when $S=R$ defines a conjugate for $\rho$, then we get a faithful left inverse (see Lemma 2.5 of [8]). If $\rho$ has $\bar{\rho}$ as conjugate, then every left inverse is of the above form. By replacing tensoring on the left by $\rho$ with the tensoring on the right by $\rho$, we can define the right inverse as well.

Definition. Let $\phi$ be the left inverse of $\rho$ defined by a solution $R, \bar{R}$ of the conjugate equations. We define the $q$-dimension $d_{q}(\rho)$ of $\rho$ by $d_{q}(\rho)=\|R\|\|\bar{R}\|$.

If we restrict to normalized solutions $R$ and $\bar{R}$, i.e. $\|R\|=\|\bar{R}\| \operatorname{implies} d_{q}(\rho)=$ $\|R\|^{2}=\left\|R^{*} \circ R\right\|$, we have $d_{q}(\rho)=R^{*} \circ R=\bar{R}^{*} \circ \bar{R}=d_{q}(\bar{\rho})$, where $d_{q}(\rho)$ is independent of the choice of $R[8]$.

We now turn to the analysis of the braiding. We start from a braiding $\theta$ and an object $\rho$ of $\mathcal{F}$ and we construct for each integer $n$ a representation of $\mathcal{B}_{\infty}$. We analyze this representation by using conjugate equations. Assume that the braiding satisfies the Hecke condition. Define the linear mappings $\Phi=\Phi_{n}:\left(\rho^{n}, \rho^{n}\right) \longrightarrow$ $\left(\rho^{n-1}, \rho^{n-1}\right)$ by $\Phi(T)=R^{*} \times 1_{\rho^{n-1}} \circ 1_{\bar{\rho}} \times T \circ R \times 1_{\rho^{n-1}}$. By using $R \in(i, \bar{\rho} \rho)$, $R^{*} \in(\bar{\rho} \rho, i), \bar{\rho}$ conjugate of $\rho, \Phi$ is just a left inverse. $\Phi$ is also a positive mapping. Now

$$
\Phi\left(1_{\rho^{n}}\right)=R^{*} \times 1_{\rho^{n-1}} \circ 1_{\bar{\rho}} \times 1_{\rho^{n}} \circ R \times 1_{\rho^{n-1}}=R^{*} \circ R \times 1_{\rho^{n-1}}
$$

and by definition of dimension $R^{*} \circ R=d_{q}(\rho)$ implies $\Phi\left(1_{\rho^{n}}\right)=d_{q}(\rho) \times 1_{\rho^{n-1}}$.

Set $\theta_{i}$ to be a generator of the infinite braid group constructed as follows:

$$
\theta_{i}=1_{i-1} \otimes \theta(\rho, \rho) \otimes 1_{N-i-1} \in\left(\rho^{N}, \rho^{N}\right), \text { for every } i=1, \ldots, N-1 .
$$

They satisfy the Hecke symmetry condition $\left(\theta_{i}-q\right)\left(\theta_{i}+1\right)=0$. We want to compute $\Phi\left(\theta(\rho, \rho) \times 1_{\rho^{n-2}}\right)$. In the symmetric case we have $\theta^{2}=1$, thus $y=\theta^{2}-1$. We assume here that $\theta$ satisfies the Hecke condition $\theta^{2}=(q-1) \theta+q 1$. Let $x \longmapsto X$ be the transformation

$$
X=2 \frac{x-q}{1+q}+1
$$

that allows us to write the braiding $\theta$ in terms of the permutation symmetry as the one in [3]. For $q$ tending to $1, X$ tends to $x$. Let us compute $\Phi\left(\theta_{(q)}\right)$, denoting for convenience by $\theta_{(q)}$ the braiding and by $\theta_{(s)}$ the permutation symmetry, i.e. in the above notation $X=\theta_{(s)}, x=\theta_{(q)}$. It turns out that

$$
\Phi\left(\theta_{q}\right)=\left(1+\frac{1-q}{1+q} d_{q}(\rho)\right) \frac{1+q}{2} .
$$

If $q \longrightarrow 1$, then $\Phi\left(\theta_{1}\right)=\Phi\left(\theta_{s}\right)$. Now the next goal is to compute $\Phi\left(\theta^{(n)}(g)\right)$, $\theta^{(n)}(g) \in\left(\rho^{n}, \rho^{n}\right)$, where $g$ is an element of the braid group. Set $g=g(p)$ with $p$ being the permutation associated to $g$. Thus

$$
\Phi\left(\theta^{(n)}(g)\right)=\Phi\left(\theta^{(n)}(g(p))\right)=\Phi\left(1_{\rho} \times \theta^{(n-1)}\left(g^{\prime}\left(p^{\prime}\right)\right)\right)
$$


if $p=1 \times p^{\prime}, p(1)=1$. This implies

$$
\Phi\left(1_{\rho} \times \theta^{(n-1)}\left(g^{\prime}\left(p^{\prime}\right)\right)\right)=d_{q}(\rho) \theta^{(n-1)}\left(g^{\prime}\left(p^{\prime}\right)\right) .
$$

If $p(1) \neq 1$, write $p=1 \times r^{\prime} \circ(12) \times 1 \circ 1 \times s^{\prime}$; then, suppressing the $g$ and writing $\theta(g(p))=\theta(p), \Phi\left(\theta^{(n)}(g(p))\right)=\theta^{(n-1)}\left(p^{\prime}\right) \cdot \Phi\left(\theta(\rho, \rho) \times 1_{\rho^{n-2}}\right)$. Here $p^{\prime}$ represents the permutation obtained from $p$ by deleting 1 from its decomposition into disjoint cycles and replacing $k$ by $k-1$ for $k \neq 1$. Compute $w^{(n)}$ by iterating $\Phi$ as $\left(\rho^{n}, \rho^{n}\right) \stackrel{\Phi}{\longrightarrow}\left(\rho^{n-1}, \rho^{n-1}\right) \stackrel{\Phi}{\longrightarrow} \ldots \stackrel{\Phi}{\longrightarrow}(i, i)$ with $w^{(n)}: \theta^{(n)}\left(\mathcal{B}_{n}\right) \longrightarrow(i, i)$, $w^{(n)}=\Phi^{(n)}=\Phi \circ \ldots \circ \Phi$.

Lemma 1. Let $g \in \mathcal{B}_{\infty}$ be an element of the infinite braid group, and let $p=$ $p(g)$ be its associated permutation, written as a product of disjoint cycles of length $k_{1}, \ldots, k_{m}$ with $k_{1}+\ldots+k_{m}=n$. Then

$$
w^{(n)}\left(\theta^{(n)}(g)\right)=\Phi^{n}\left(\theta^{(n)}(g)\right)=\left(d_{q}(\rho)\right)^{m} \Phi\left(\theta(\rho, \rho) \times 1_{\rho^{n-2}}\right)^{m}
$$

where $\Phi\left(\theta(\rho, \rho) \times 1_{\rho^{n-2}}\right)$ is given by $(5)$.

Proof. The proof follows from the formula for $\Phi\left(\theta^{(n)}(g)\right)$ where $\Phi\left(1_{\rho}\right)=d_{q}(\rho)$.

Our goal is to prove that $w^{(n)}$ is a faithful trace and hence to determine the $\mathrm{C}^{*}$-algebra generated by $\theta^{(n)}(g)$.

Lemma 2. If $d_{q}(\rho)=R^{*} \circ R$ is the $q$-dimension, then

$$
d_{q}(\rho)\left\|\Phi\left(T^{*} \circ T\right)\right\| \geq\|T\|^{2}, \quad T \in\left(\rho^{n}, \rho^{n}\right) .
$$

Proof. First we need to have an equation involving $\left(T^{*} \circ T\right) \times 1_{\rho}$ and $1_{\rho} \times\left(T^{*} \circ T\right)$. We know that the braiding satisfies $\theta\left(\sigma, \sigma^{\prime}\right) \circ R \times R^{\prime}=R^{\prime} \times R \circ \theta\left(\rho, \rho^{\prime}\right)$ if $R \in(\rho, \sigma)$, $R^{\prime} \in\left(\rho^{\prime}, \sigma^{\prime}\right), \theta\left(\sigma, \sigma^{\prime}\right) \in\left(\sigma \sigma^{\prime}, \sigma^{\prime} \sigma\right) \theta\left(\rho, \rho^{\prime}\right) \in\left(\rho \rho^{\prime}, \sigma \sigma^{\prime}\right)$.

Let us compute

$$
\theta\left(\rho, \rho^{n}\right) \circ 1_{\rho} \times T^{*} \circ T \circ \theta\left(\rho^{n}, \rho\right)
$$

when $\theta$ is the braiding, actually a Hecke symmetry. By using the notation $\theta_{(s)}$ and $\theta_{(q)}$ introduced earlier, we have that (6) can be rewritten as

$$
\theta_{(q)}\left(\rho, \rho^{N}\right)^{2} \circ\left(T^{*} \circ T \times 1_{\rho}\right)=\left(\left(\frac{1+q}{2}\right) \theta_{(s)}+\left(\frac{q-1}{2}\right)\right)^{2} \circ\left(T^{*} \circ T \times 1_{\rho}\right) .
$$

Taking $1_{\rho} \times T \circ \theta_{q}\left(\rho^{n}, \rho\right)$ instead of $T$ in $d_{q}(\rho) \Phi\left(T^{*} \circ T\right) \geq \Phi\left(T^{*}\right) \Phi(T)$ (see (2.7) of [8]), by using (7) together with $\Phi\left(1_{\rho} \times S \circ S^{\prime} \circ 1_{\rho} \times S^{\prime \prime}\right)=S \circ \Phi\left(S^{\prime}\right) \circ S^{\prime \prime}$ and (4) we get

$$
\begin{aligned}
d_{q}(\rho) \Phi & \left(\left(1_{\rho} \times T \circ \theta_{q}\left(\rho^{n}, \rho\right)\right)^{*}\left(1_{\rho} \times T \circ \theta_{q}\left(\rho^{n}, \rho\right)\right)\right) \\
\geq & \theta_{q}\left(\rho, \rho^{n-1}\right) \circ \Phi\left(\theta_{q}(\rho, \rho) \times 1_{\rho^{n-1}}\right) \\
& \circ T^{*} \circ T \circ \Phi\left(\theta_{q}(\rho, \rho) \times 1_{\rho^{n-1}} \circ \theta_{q}\left(\rho^{n-1}, \rho\right)\right) .
\end{aligned}
$$

Taking norms we get

$$
\begin{aligned}
d_{q}(\rho) & \left\|\Phi\left(\left(1_{\rho} \times T \circ \theta_{q}\left(\rho^{n}, \rho\right)\right)^{*}\right) \circ\left(1_{\rho} \times T \circ \theta_{q}\left(\rho^{n}, \rho\right)\right)\right\| \\
\geq & \| \theta_{q}\left(\rho, \rho^{n-1}\right) \circ \Phi\left(\theta_{q}(\rho, \rho) \times 1_{\rho^{n-1}}\right) \\
& \circ T^{*} \circ T \circ \Phi\left(\theta_{q}(\rho, \rho) \times 1_{\rho^{n-1}} \circ \theta_{q}\left(\rho^{n-1}, \rho\right)\right) \| .
\end{aligned}
$$


If $\Phi\left(T^{*} \circ T\right)=0$, the left hand side gives 0 and implies $\|\tilde{T}\|^{2}=0$, where $\tilde{T}=$ $T \circ \theta_{q}\left(\rho, \rho^{n-1}\right)$; this implies $T=0$. Thus $\Phi$ is faithful.

Lemma 3. If the $q$-dimension $d_{q}(\rho)^{-1} \Phi(\theta(\rho, \rho))=q^{N}[N]_{q}^{-1}$, then the $C^{*}$-algebra generated by $\theta(g)$ is canonically isomorphic to the one generated by the representation of $\mathcal{B}_{\infty}$ given by the Markov trace of modulus $z=q^{N}\left([N]_{q}\right)^{-1}$ on a Hilbert space of finite dimension $N$.

Proof. Just check that $w^{(n)}\left(\theta_{q}(g)\right)$ gives the Markov trace of the specified representation of $\mathcal{B}_{\infty}$ as found in Lemma 1.4 of [1].

Let us introduce the concept of determinant of an object, which can be called the $q$-determinant, because of the braiding defining it. Given $n$ and $\rho$ denote by $A_{n}^{\rho}$ the totally $q$-antisymmetric projection in $\left(\rho^{n}, \rho^{n}\right)$ of the form

$$
A_{n}^{\rho}=\left([n]_{q^{-1}} !\right) \sum_{\sigma \in S_{n}}(-q)^{-l(\sigma)} \theta(g(\sigma)) .
$$

Let $\rho$ be an object of $\operatorname{rank} N$ in the sense that $A_{N}^{\rho} \neq 0, A_{k}^{\rho}=0, \forall k>N$. We say that a pair $(\gamma, V)$ consisting of an object $\gamma$ and an isometry $V \in\left(\gamma, \rho^{N}\right)$ with $V \circ V^{*}=A_{N}^{\rho}$ defines a $q$-determinant for $\rho$. This agrees with the well known formula as in [1], [16] for the totally $q$-antisymmetric projection in $\left(\rho^{n}, \rho^{n}\right)$, where $\rho=H$, the Hilbert space of dimension $N$.

Let us assume from now on that the strict braided tensor $\mathrm{C}^{*}$-category with conjugates $(\mathcal{F}, \theta)$ is generated by a single object $\rho \in E n d \mathcal{A}$, where $\mathcal{A}$ is a $\mathrm{C}^{*}$-algebra, arrows are linear mappings in $\left(\rho^{r}, \rho^{s}\right), \rho^{r}=\rho \otimes \ldots \otimes \rho(r$ times $)$. The braiding $\theta$ is defined as follows: $\theta(\rho, \rho) \in\left(\rho^{2}, \rho^{2}\right), \theta_{i}=1_{i-1} \otimes \theta(\rho, \rho) \otimes 1_{n-i-1} \in\left(\rho^{n}, \rho^{n}\right)$, for every $i=1, \ldots, n-1$; by shifting the generator $\theta(\rho, \rho)$ we get all the generators of $\mathcal{B}_{\infty}$, with the following relations:

1. $\theta_{i} \theta_{j}=\theta_{j} \theta_{i}$, for $|i-j|>1$;

2. $\theta_{i} \theta_{i+1} \theta_{i}=\theta_{i+1} \theta_{i} \theta_{i+1}$, for $1 \leq i \leq n-1$;

3. $\left(\theta_{i}-q\right)\left(\theta_{i}+1\right)=0$ for $1 \leq i<n$, i.e. the Hecke symmetry condition.

\section{Hecke symmetries and actions on extended Cuntz algebras}

We consider $\left(\mathcal{F}_{\rho}, \theta\right)$ to be a braided tensor $\mathrm{C}^{*}$-category as at the end of section 3. Suppose $\theta\left(g_{0}\right) \in\left(\rho^{2}, \rho^{2}\right) ; g_{0} \in \mathcal{B}_{\infty}$ is the specified generator and $g_{i}=\sigma^{i}\left(g_{0}\right)$. If $\theta\left(g_{0}\right)$ is a Hecke symmetry we say that the endomorphism $\rho$ has a Hecke symmetry if (1)-(3) above are satisfied. Suppose that there exists a determinant, i.e. $V \in\left(i, \rho^{N}\right)$ such that $V \circ V^{*}=A_{\rho}^{N}$. Let $\hat{\rho}$ be the endomorphism which arises by tensoring on the left with $1_{\rho}$.

Lemma 4. If $(i, V)$ is a determinant for an object $\rho$, then $V^{*} \times 1_{\rho} \circ 1_{\rho} \times V=$ $(-1)^{N-1} d_{q}(\rho)^{-1} \lambda_{q} I_{\rho}$, where $\lambda_{q}=\Phi(\theta(\rho, \rho))$.

Proof. Let $S=\theta\left(\rho, \rho^{\prime}\right) \circ \bar{S}$, given by

$$
S=\theta\left(\rho, \rho^{\prime}\right) \circ 1_{\rho} \times W^{*} \circ V d_{q}(\rho)^{1 / 2}=W^{*} \times 1_{\rho} \circ \theta\left(\rho, \rho^{N-1}\right) \circ V d_{q}(\rho)^{1 / 2} .
$$


Thus $S^{*} \times 1_{\rho} \circ 1_{\rho^{\prime}} \times \theta(\rho, \rho) \circ S \times 1_{\rho}=\Phi(\theta(\rho, \rho))$. Take $\tilde{S}=\lambda_{q}^{-1 / 2} S$. As in Lemma 3.6 of [3], this defines a conjugate $\rho^{\prime}$ for $\rho$. Then

$$
\begin{gathered}
1_{\rho}=\lambda_{q}^{-1} d_{q}(\rho) V^{*} \times 1_{\rho} \circ \theta\left(\rho^{N-1}, \rho\right) \times 1_{\rho} \circ W \times 1_{\rho^{2}} \circ 1_{\rho^{\prime}} \circ \theta(\rho, \rho) \\
\circ W^{*} \times 1_{\rho^{2}} \circ \theta\left(\rho, \rho^{N-1}\right) \times 1_{\rho} \circ V \times 1_{\rho} \\
=\lambda_{q}^{-1} d_{q}(\rho) V^{*} \times 1_{\rho} \circ \theta\left(\rho^{N-1}, \rho\right) \times 1_{\rho} \circ 1_{\rho} \circ 1_{\rho^{N-1}} \times \theta(\rho, \rho) \circ \theta\left(\rho, \rho^{N-1}\right) \\
\times 1_{\rho} \circ 1_{\rho} \times A_{N-1}^{\rho} \times 1_{\rho} \circ V \times 1_{\rho},
\end{gathered}
$$

since $V \circ V^{*}=A_{N}^{\rho}, 1_{\rho} \times A_{N-1}^{\rho} \circ V=V$. Furthermore

$$
\theta\left(\rho^{N-1}, \rho\right) \times 1_{\rho} \circ 1_{\rho^{N-1}} \times \theta(\rho, \rho)=\theta\left(\rho^{N}, \rho\right), \quad \theta\left(\rho, \rho^{N-1}\right) \circ V=(-1)^{N-1} V .
$$

Thus

$$
\begin{aligned}
1_{\rho} & =(-1)^{N-1} \lambda_{q}^{-1} d_{q}(\rho) V^{*} \times 1_{\rho} \circ \theta\left(\rho^{N}, \rho\right) \circ V \times 1_{\rho} \\
& =(-1)^{N-1} \lambda_{q}^{-1} d_{q}(\rho) V^{*} \times 1_{\rho} \circ 1_{\rho} \times V .
\end{aligned}
$$

Remark. Let $\rho$ be an object such that $d_{q}(\rho) \neq 0$. Let $\hat{\rho}$ be the endomorphism of $O_{\rho}$ corresponding to the tensoring on the left with $1_{\rho}$, in $\mathcal{F}_{\rho}, \hat{\rho}(i(T))=i(1 \otimes T)$. Now, we have $O_{\rho}^{k} \subset[\hat{\rho}]^{k}$ because of the existence of the ${ }^{*}$-functor $i: \mathcal{F} \longrightarrow E n d O_{\rho}$. By Lemma 4 we have an isometry $V \in\left(i, \rho^{N}\right)$ such that $V^{*} \times 1_{\rho} \circ 1_{\rho} \times V=$ $(-1)^{N-1} d_{q}(\rho)^{-1} \lambda_{q}$ so there exists $S_{m} \in\left(i, \hat{\rho}^{m N^{m}}\right)$ such that $S_{m}^{*} \hat{\rho}^{m}\left(S_{m}\right)=$ $(-1)^{N^{m}-1} d_{q}(\rho)^{-m} \lambda_{q}^{m} 1$; this implies there is an action of $\mathbf{T}$ on $[\hat{\rho}]=O_{\rho}$ determining the grading, by Lemma 5.12 of [8]. This action agrees on a dense $*_{\text {-subalgebra }}$ with the action determining the grading of $O_{\rho}$. Hence the two actions agree so that $[\hat{\rho}]^{k}=O_{\rho}^{k}$.

Corollary. Let $\rho$ be a special object with $q$-dimension $d_{q}(\rho)$. Then the representation $\theta_{\rho}$ of (3) defines a Hecke symmetry for the endomorphism $\hat{\rho}$ of $O_{\rho}$.

Proof. Suppose $X \in \bigcup_{r, r+k \geq 0} i\left(\rho^{r}, \rho^{k+r}\right)$; then for $r$ sufficiently large we have

$$
\theta_{\rho}(r+k, 1) X=\hat{\rho}(X) \theta_{\rho}(r, 1) .
$$

Now $q^{-1}\left(\theta_{\rho}(r+k, 1) X \hat{\theta}_{\rho}(1, r)\right)=\hat{\rho}(X)$, where $\hat{\theta}_{\rho}(1, r)=\left(\theta_{\rho}(1, r)-(q-1)\right)$.

Such elements $X$ are dense in $O_{\rho}^{k}$, hence we have

$$
\hat{\rho}(X)=\lim _{s \longrightarrow \infty} \theta_{\rho}(s+k, 1) X \tilde{\theta}_{\rho}(1, s) .
$$

We need to prove that (8) holds for $X \in\left(\hat{\rho}^{r}, \hat{\rho}^{r+k}\right)$. If $X \in\left(\hat{\rho}^{r}, \hat{\rho}^{r+k}\right)$, then by the above remark, $X \in O_{\rho}^{k}$ but $\theta_{\rho}(1, r)=\hat{\rho}^{r-1}\left(\theta_{\rho}\right) \ldots \hat{\rho}\left(\theta_{\rho}\right) \theta_{\rho}$, hence

$$
\theta_{\rho}(s+k, 1) X \tilde{\theta}_{\rho}(1, s)=\theta_{\rho} \hat{\rho}\left(\theta_{\rho}\right) \ldots \hat{\rho}^{s+k-1}\left(\theta_{\rho}\right) X \hat{\rho}^{s-1}\left(\tilde{\theta}_{\rho}\right) \ldots \hat{\rho}\left(\tilde{\theta}_{\rho}\right) \tilde{\theta}_{\rho}
$$

and if $s \geq r$ the latter however is equal to

$$
\theta_{\rho} \hat{\rho}\left(\theta_{\rho}\right) \ldots \hat{\rho}^{r+k-1}\left(\theta_{\rho}\right) X \hat{\rho}^{r-1}\left(\tilde{\theta}_{\rho}\right) \ldots \hat{\rho}\left(\tilde{\theta}_{\rho}\right) \tilde{\theta}_{\rho} .
$$

Taking limits we verify $(8)$ :

$$
\hat{\rho}(X)=\lim _{s \longrightarrow \infty} \theta_{\rho}(s+k, 1) X \tilde{\theta}_{\rho}(1, s)=\theta_{\rho}(r+k, 1) X \tilde{\theta}_{\rho}(1, r) .
$$


Let $\theta_{q}$ be a Hecke symmetry of rank $N$ on a Hilbert space $\mathcal{H}$ of dimension $N$. Then by Theorem 3.1 of [1] there is associated a compact matrix quantum group $\left(A\left(\theta_{q}\right), u\right)$ given by $A\left(\theta_{q}\right)=C^{*}\left\langle\left(u_{i j}\right)_{i, j=1, \ldots, N}: u\right.$ unitary, $\left.u^{\otimes N} \xi=\xi\right\rangle$, where $\xi$ is a unit vector in $\operatorname{Im} P_{N}$, where $\operatorname{Im} P_{N}$ is the $q$-analogue of the space of antisymmetric tensors in $\mathcal{H}^{\otimes N}$ and $S_{q}=\left([N]_{q^{-1}} !\right)^{-1} \sum_{\sigma \in S_{N}}(-q)^{-l(\sigma)} T_{\sigma}$.

Theorem. Let $g \longrightarrow \theta_{\rho}(g)$ be a Hecke symmetry for an endomorphism $\hat{\rho}$ of the $C^{*}$-algebra $O_{\rho}$ and suppose there is $R \in i\left(i, \rho^{N}\right)$ with

$$
R^{*} \circ \hat{\rho}(R)=(-1)^{N-1}[N]_{q}^{-1} q^{N}, \quad R \circ R^{*}=\left([N]_{q^{-1}} !\right)^{-1} \sum_{\sigma \in S_{N}}(-q)^{-l(\sigma)} \theta_{\rho}(g) .
$$

Then there is a unique ${ }^{*}$-monomorphism $\mu:\left(O_{N}\right)^{\Gamma} \longrightarrow O_{\rho}$, with $\mu\left(\theta_{q}\right)=\theta_{\rho}$, $\mu\left(S_{q}\right)=R, \mu \circ \sigma=\hat{\rho} \circ \mu$. As in [10], $\sigma$ denotes the canonical endomorphism of $O_{N}$ and $\Gamma$ is the coaction of the compact matrix quantum group $A\left(\theta_{q}\right)$ on $O_{N}$.

Proof. By the corollary the representation $g \longmapsto \theta_{\rho}(g)$ has a Hecke symmetry for $\hat{\rho}$, such that $\theta_{\rho}\left(g_{s+1}\right) X=\hat{\rho}(X) \theta_{\rho}\left(g_{r+1}\right)$ for every $X \in\left(\hat{\rho}^{r}, \hat{\rho}^{s}\right)$. There exists $R \in i\left(i, \rho^{N}\right)$ such that $R^{*} \hat{\rho}(R)=(-1)^{-N}[N]_{q}^{-1} q^{N}$, and there exists $A_{\rho}^{N}$ such that $A_{\rho}^{N}=R \circ R^{*}$. Setting $\Phi(A)=\bar{R}^{*} \times 1_{\hat{\rho}} \circ 1_{\hat{\rho}^{N-1}} \circ A \circ \bar{R} \times 1_{\hat{\rho}}, A \in O_{\rho}, \Phi$ defines a left inverse for $\hat{\rho}$, by using the conjugates $\bar{R} \in\left(i, \hat{\rho}^{N-1} \hat{\rho}\right), \bar{R}^{*} \in\left(\hat{\rho}^{N-1} \hat{\rho}, i\right)$, as in Lemma 4. By direct computation, $\Phi\left(\theta_{\rho}(g)\right)=\lambda_{q} d_{q}(\rho)^{-1}$. By Lemma 3, $\Phi^{(n)}\left(\theta_{\rho}^{(n)}(g)\right)$ gives a Markov trace of modulus $q^{N}[N]_{q}^{-1}$ and the $\mathrm{C}^{*}$-algebra $C^{*}\left(\theta_{\rho}(g)\right)$ is the one generated by the representation given by the Markov trace on the finite-dimensional Hilbert space. Then $A_{\rho}^{k}=0$, for every $k>N$. Denote by $\theta_{q}$ the Hecke symmetry on a finite-dimensional Hilbert space [1]; hence there exists a universal compact matrix quantum group $A\left(\theta_{q}(g)\right)$ given as $C^{*}\left(u, u^{\otimes N} \xi=\xi, \xi \in \operatorname{Im}\left(P_{N}\right)\right)$ and by $[10]$ there exists a natural coaction $\Gamma: O_{N} \longrightarrow A\left(\theta_{q}(g)\right) \otimes O_{N}$, such that $\left(O_{N}\right)^{\Gamma}$, the fixed point algebra under $\Gamma$, is the $\mathrm{C}^{*}$-algebra generated by $\theta_{q}(g)$. Apply the main theorem of [9] and the result follows.

\section{ACKNOWLEDGEMENTS}

The author thanks Professors E. C. Lance and P. E. T. Jorgensen for useful discussions, and the Engineering and Physical Sciences Research Council of the UK government for the support of this research.

\section{REFERENCES}

[1] T. Banica, (1996), The quantization of $C(S U(N))$ and some related amenability questions from the fusion semiring viewpoint, preprint, Marseille.

[2] J. Cuntz, (1977) Simple C*-algebras generated by isometries, Comm. Math. Phys. 57, p. 173-185. MR 57:7189

[3] S. Doplicher, J.E. Roberts, (1989), A new duality theory for compact groups, Invent. math., 98, 157-218. MR 90k:22005

[4] S. Doplicher, J.E. Roberts, (1987), Duals of compact Lie groups realized in the Cuntz algebras, J. Functional Analysis, 74, 90-120. MR 98a:22011

[5] V.F.R. Jones, (1987), Hecke algebra representations of braid groups and link polynomials, Annals of Mathematics, 126, p. 335-388. MR 89c:46092

[6] A. Joyal, R. Street, (1986) Braided monoidal categories, Macquarie Mathematics report n. 860081.

[7] C. Kassel, (1994), Quantum Groups, Springer Verlag. MR 96:17041 
[8] R. Longo, J. E. Roberts, (1995), Theory of dimension, preprint, Rome.

[9] A. Paolucci, (1996), Remarks on braided $\mathrm{C}^{*}$-categories and endomorphisms of $\mathrm{C}^{*}$-algebras, J. Operator Theory, 36, p. 157-177. MR 97m:46113

[10] A. Paolucci, (1997), Coactions of Hopf Algebras on Cuntz algebras and their fixed point algebras, Proc. Amer. Math. Soc., 125, p. 1033-1042. MR 97f:46106

[11] S.L. Woronowicz, (1987), Compact matrix pseudogroups, Comm. Math. Phys. 111, 613-665. MR 88m:46079

[12] S.L. Woronowicz, (1987), Twisted SU(2) group. An example of a non commutative differential calculus, Publ. RIMS 23, 117-181.

[13] S.L. Woronowicz, (1988), Tannaka-Krein duality for compact matrix pseudogroups, twisted $S U(N)$ groups, Inventiones Math., 93, p. 35-76. MR 90e:22033

[14] S.L. Woronowicz, (1993), Compact Quantum Group, preprint, Warsaw.

[15] D.N. Yetter, (1990), Quantum groups and representations of monoidal categories, Math. Proceedings Camb. Phil. Soc. 108, p. 261-290. MR 91k:16028

[16] H. Wenzl, (1988), Hecke algebras of type $A_{n}$ and subfactors, Invent. Math. 92, p. 349-383. MR 90b: 46118

School of Mathematics, University of Leeds, LS2 9JT, United Kingdom

Current address: Dipartimento di Matematica, Università di Torino, via Carlo Alberto, 10, 10124 Torino, Italy

E-mail address: paolucci@dm.unito.it 